\title{
Radiation Dosimetry of 3D Rotational Neuroangiography and 2D-DSA in Children
}

\author{
(D) N.A. Shkumat, DM.M. Shroff, and (DP. Muthusami
}

\begin{abstract}
BACKGROUND AND PURPOSE: The benefit-risk assessment concerning radiation use in pediatric neuroangiography requires an extensive understanding of the doses delivered. This work evaluated the effective dose of 3D rotational angiography in a cohort of pediatric patients with complex neurovascular lesions and directly compared it with conventional 2D-biplane DSA.
\end{abstract}

MATERIALS AND METHODS: Thirty-three 3D rotational angiography acquisitions were acquired in 24 pediatric patients (mean age, 10.4 years). When clinically indicated, following 2D-biplane DSA, 3D rotational angiography was performed with 1 of 3 technical protocols (2 subtracted, 1 unsubtracted). The protocols consisted of 1 factory and 2 customized techniques, with images subsequently reconstructed into CT volumes for clinical management. Raw projections and quantitative dose metrics were evaluated, and the effective dose was calculated.

RESULTS: All 3D rotational angiography acquisitions were of diagnostic quality and assisted in patient management. The mean effective doses were $0.5,0.12$, and $0.06 \mathrm{mSv}$ for the factory-subtracted, customized-subtracted, and customized-unsubtracted protocols, respectively. The mean effective dose for 2D-biplane DSA was $0.9 \mathrm{mSv}$. A direct intraprocedural comparison between 3D and 2D acquisitions indicated that customized 3D rotational angiography protocols delivered mean relative doses of $9 \%$ and $15 \%$ in unsubtracted and subtracted acquisitions, respectively, compared with biplane DSA, whereas the factory subtracted protocol delivered $68 \%$.

CONCLUSIONS: In pediatric neuroangiography, the effective dose for 3D rotational angiography can be significantly lower than for 2D-biplane DSA and can be an essential adjunct in the evaluation of neurovascular lesions. Additionally, available 3D rotational angiography protocols have significant room to be tailored for effectiveness and dose optimization, depending on the clinical question.

ABBREVIATIONS: $3 \mathrm{DRA}=3 \mathrm{D}$ rotational angiography; $\mathrm{K}_{\mathrm{a}, \mathrm{r}}=$ reference point air kerma

A ngiography for the delineation of pediatric intracerebral vascular lesions can often require digital subtraction angiography at multiple angles for complete anatomic and hemodynamic assessment. To supplement existing technology, the use of 3D rotational angiography (3DRA) has increased in recent years and is now a viable imaging option available to the neuroradiologist. $^{1-4}$ It promises to add relevant clinical information that can increase diagnostic confidence and potentially decrease the amount of fluorography while allowing improved and more efficient intervention planning. Existing applications of 3DRA in

Received October 4, 2017; accepted after revision December 28.

From the Department of Diagnostic Imaging (N.A.S., M.M.S., P.M.), The Hospital for Sick Children, Toronto, Ontario, Canada; and Department of Medical Imaging (N.A.S., M.M.S., P.M.), University of Toronto, Toronto, Ontario, Canada.

Please address correspondence to Nicholas A. Shkumat, Department of Diagnostic Imaging, The Hospital for Sick Children, 555 University Ave, Suite 2175A

Toronto, ON, Canada M5G 1X8; e-mail: nicholas.shkumat@sickkids.ca

http://dx.doi.org/10.3174/ajnr.A5568 neuroangiography range from assessing the anatomy of arteriovenous malformations, volume-rendered vessel reformats for aneurysm treatment, CT reconstruction for hemorrhage and multiplanar reformation, and MR imaging fusion for presurgical evaluation and guidance. ${ }^{5-8}$

Although the concept of dose reduction and optimization is often described in pediatric literature, the comparison of the radiation dose between individual imaging events within a procedure is not often evaluated. ${ }^{9,10}$ This practice is due to a number of factors, including the inherent variability of neuroangiographic procedures, the complexity of lesions being assessed, a wide range of body habitus, differences in technical protocols between vendors and models, operator preferences, and lack of viable patient reference models/phantoms. Previous research has evaluated the radiation dose in pediatric neuroangiography with respect to readily available, system-provided dose surrogates, though the work was not extended to effective dose, which is widely accepted as a general quantifier of radiation risk. ${ }^{7}$ The correlation between 
these reference metrics and organ or effective dose is weak and has not been published for the pediatric population. ${ }^{11,12}$ The effective dose for pediatric 3DRA has been investigated for applications in radiation oncology and cardiology and in adult patients and phantoms. ${ }^{13-17}$ Although effective dose is an imperfect metric and not intended for use in individual patients, it can be a useful and valuable tool for the identification, assessment, and comparison of radiation burden in similar imaging scenarios. It is also an effective method of radiation dose communication throughout radiology and associated medical specialties.

With increasing use of 3DRA and advanced rotational imaging techniques, this work sought to calculate and compare the effective dose of clinical standard-of-care 2D-biplane DSA and various 3DRA protocols implemented at our institution. This research will aid in the continual assessment of the benefit-risk ratio with each application of ionizing radiation in the complex pediatric neurovascular environment.

\section{MATERIALS AND METHODS}

Within an 18-month period, thirty-three 3D-acquisitions were performed in 24 patients requiring conventional angiography. All procedures were performed by 2 pediatric neuroradiologists (P.M. and M.M.S.) in a biplane interventional suite (Artis Q biplane; Siemens, Erlangen, Germany).

Written informed consent was obtained from parents/guardians for all procedures.

All procedures were performed with the patient under general anesthesia. Patients were catheterized via femoral artery access. Injections of iodinated contrast were delivered through a power injector, with flow rates ranging between 2.5 and $5 \mathrm{~mL} / \mathrm{s}$ and total volumes ranging from 6 to $10 \mathrm{~mL}$. The clinical indications for these studies are described in Table 1.

\section{Imaging}

2D-DSA images were obtained using a 32- to $42-\mathrm{cm}$ FOV with an acquisition rate of 3-6 frames/s. Acquisition length varied depending on the indication and individual hemodynamics.

Customized technical protocols were used with a target kilovolt(peak) of 70, a requested detector dose of $1200 \mathrm{nGy} /$ frame, and applied copper filtration between 0.1 and $0.3 \mathrm{~mm}$. Postprocessing parameters varied depending on the imaging task.

Table 1: Patient demographics and indications

\begin{tabular}{lc}
\multicolumn{1}{c}{ Parameter } & Value \\
\hline Age (mean) (yr) & $10.4 \pm 5.1$ \\
Male/female & $18: 6$ \\
Procedure indication (No.) & \\
AVM & 28 \\
Intra-arterial chemotherapy & 2 \\
Aneurysm & 1 \\
Aberrant neurovasculature & 1 \\
Presurgical embolization & 1 \\
\hline
\end{tabular}

Following biplane imaging, a 5-second 3DRA was acquired with the patient at the isocenter and the FOV fixed at $42 \mathrm{~cm}$. Three 3DRA protocols were evaluated in this article: a customized unsubtracted protocol described in previous work and 2 subtracted protocols, before and after customization. ${ }^{7}$ Pathology dictated the decision to acquire subtracted or unsubtracted imaging volumes. Subtracted 3DRA is composed of 2 rotations, the first creating a "mask" volume, with the second in close succession using iodine contrast material. Together, they are referred to as the subtracted acquisition. Unsubtracted acquisitions use a single rotational volume with contrast. Select technical specifications are described in Table 2.

\section{Dosimetric Analysis}

Effective dose was calculated through a multistep process. Quantitative radiation dose surrogates were extracted through assessment of radiation dose structured reports and information parsed from each 3DRA imaging volume. Validation of integrated radiation output systems was made with an external radiation dosimeter following the methodology specified in the American Association of Physicists in Medicine Task Group Report 190. ${ }^{17}$

Details of each projection for both 2D-DSA and 3DRA were obtained, including the following: kilovolt(peak), filtration, tube current, exposure time, source-imager distance, projection angulation, cranial-caudal angulation, milliampere, pulse width, table position, air kerma area product, and reference point air kerma $\left(\mathrm{K}_{\mathrm{a}, \mathrm{r}}\right)$. Attenuation of the imaging table and mattress for projections with complete interception of the FOV (namely, posterioranterior and small angles) was accounted for in the assessment. Oblique projections with partial table attenuation were conservatively assumed to be unattenuated.

The representative effective dose was determined through Monte Carlo modeling software (PCXMC 2.0; STUK, Helsinki, Finland). The method applied was a simple application of photon transport based on stochastic simulation of interactions between $\mathrm{x}$-rays and matter and the deposition of energy to organs within the imaged volume. ${ }^{18}$ Mathematic hermaphrodite phantoms of 4 reference ages and sizes were used $(1,5,10$, and 15 years $)$ to simulate each patient in this study. The age of the reference model closest to true patient age was used for each calculation.

For biplane 2D-DSA acquisitions, patient entrance air kerma was determined for precise angles (projection and craniocaudal angulation) of each stationary fluorographic run. These data with corresponding beam quality and geometric considerations, including source-to-entrance distances, were applied through the Monte Carlo simulation process to calculate the effective dose for individual posteroanterior and lateral runs. For display and discussion, each acquisition pair was summed to provide the total biplane 2D-DSA effective dose.

For 3DRA acquisitions, we determined the patient entrance air

Table 2: Technical factors for 3DRA acquisition protocols

\begin{tabular}{lccccccc}
\hline & $\begin{array}{c}\text { Projections per } \\
\text { Rotation }\end{array}$ & Rotation & $\begin{array}{c}\text { Rotations Required } \\
\text { per Acquisition }\end{array}$ & $\begin{array}{c}\text { kVp } \\
\text { Target }\end{array}$ & $\begin{array}{c}\text { Detector Dose } \\
\text { (nGy/projection) }\end{array}$ & $\begin{array}{c}\text { Filtration } \\
\text { (mm Cu) }\end{array}$ & $\begin{array}{c}\text { Half-Value } \\
\text { Layer (mm Al) }\end{array}$ \\
\hline Protocol 1 (subtracted) & 133 & $200^{\circ}$ & 2 (mask, contrast) & 70 & 360 & 0.0 & 2.9 \\
Protocol 2 (subtracted) & 133 & $200^{\circ}$ & 2 (mask, contrast) & 90 & 200 & 0.3 & 7.1 \\
Protocol 3 (unsubtracted) & 248 & $200^{\circ}$ & 1 (contrast) & 90 & 100 & 0.6 & 8.2 \\
\hline
\end{tabular}




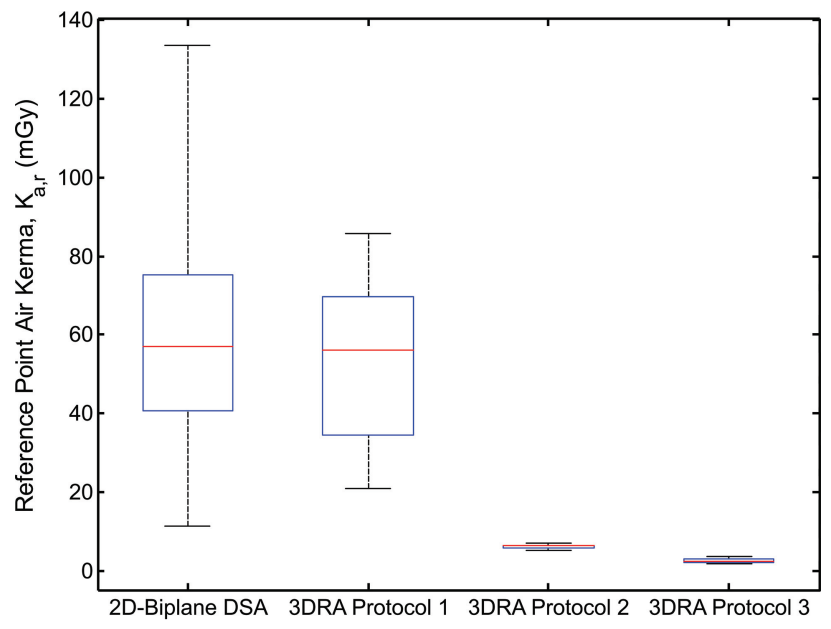

FIG 1. Box-and-whisker plot depicting the reference point air kerma of biplane DSA and 3DRA protocols across all patients. Boxes denote median, lower, and upper quartiles, with whiskers capturing values outside the interquartile range.

kerma for each angulation, individually accounting for changes in tube output per projection and correcting for source-to-entrance distances for each representative patient age and size. Beam collimation and FOV were assessed for each 3DRA volume and propagated into the Monte Carlo calculation model. Communication of air kerma, absorbed dose, and effective dose during each 3D acquisition indicated the summation across all projections acquired across the angular range of each rotation and the number of rotations required for the 3DRA (2 for subtracted, 1 for unsubtracted).

In all calculations, the latest tissue-weighting factors from the International Commission on Radiologic Protection Report 103 were applied in the calculation of effective dose. ${ }^{19}$ Following the calculations for biplane 2D-DSA and 3DRA in the same vessel and patient, relative comparisons were made for direct assessment.

\section{RESULTS}

Of the 33 3DRA acquisitions in this work, 12 were acquired with the conventional 3D-DSA (subtracted) protocol (referred to as protocol 1); 6, with the modified 3D-DSA (subtracted) protocol (protocol 2); and 15, with the unsubtracted protocol (protocol 3). The integrated system dosimeter in both planes was confirmed to be accurate to within $6 \%$ through external validation with a calibrated dosimetry system.

The primary quantitative radiation dose surrogate, $K_{a, r}$ for aggregate 2D-DSA and 3DRA acquisitions, is shown in Fig 1. As evaluated in prior work, a large range is observed in biplane 2D DSA runs. ${ }^{7}$ The mean and median $\mathrm{K}_{\mathrm{a}, \mathrm{r}}$ for $2 \mathrm{D}$-biplane DSA were 60.1 and $56.9 \mathrm{mGy}$, respectively, with a total range of 11.5-133.5 mGy. When we evaluated 3D protocols, the mean and median air kerma for the subtracted acquisition were 52.8 and $56.0 \mathrm{mGy}$, with a range of $21.0-85.6 \mathrm{mGy}$ (factory $3 \mathrm{D}$-acquisition, protocol 1) and 6.4 and $6.6 \mathrm{mGy}$ with a range of 5.2-7.3 $\mathrm{mGy}$ for the customized 3D-DSA acquisition (protocol 2). For unsubtracted $3 \mathrm{D}$ acquisitions (protocol 3), the mean and median air kerma were 2.7 and $2.6 \mathrm{mGy}$, with range of $1.9-3.7 \mathrm{mGy}$.

Figure 2 illustrates the considerable difference in reference

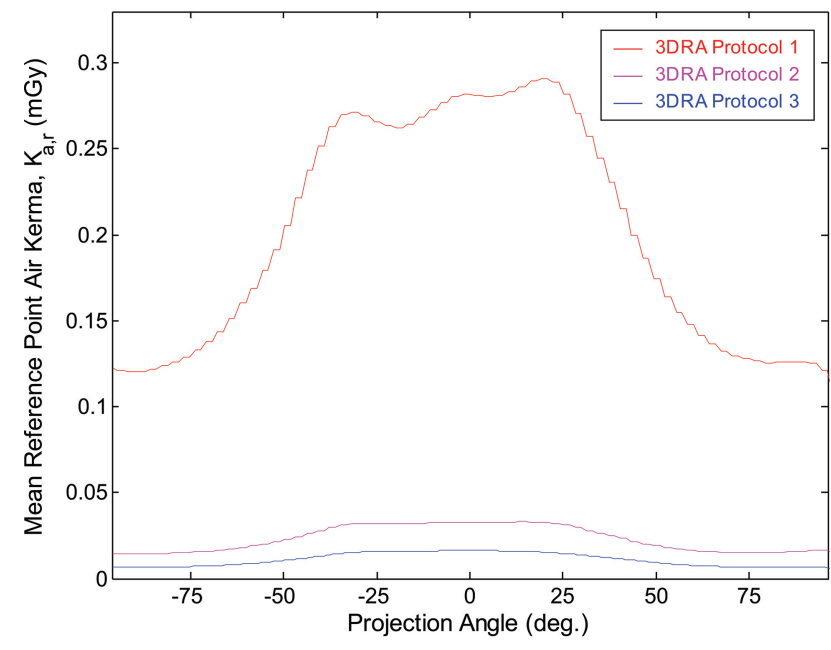

FIG 2. Mean reference point air kerma as a function of projection angle for 3DRA protocols 1, 2, and 3 .

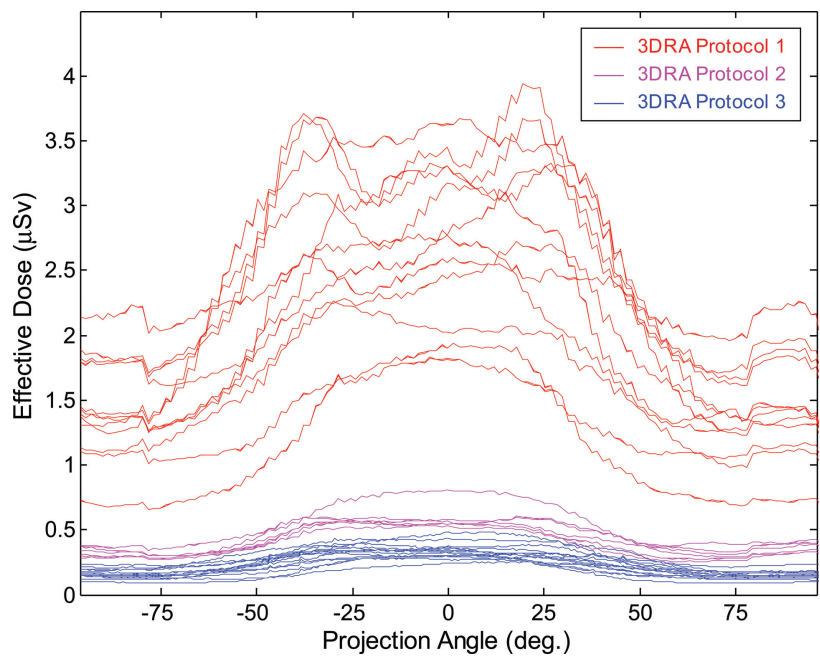

FIG 3. Effective dose as a function of projection angle for 3DRA protocols 1, 2, and 3. Each line indicates the effective dose for an individual rotation.

point air kerma as the tube and detector rotate about the patient in 3DRA. Each data point corresponds to the mean $\mathrm{K}_{\mathrm{a}, \mathrm{r}}$ across all patients, acquired using protocols 1,2 , and 3. The deviation in air kerma around the $200^{\circ}$ rotation is a function of the nonuniform attenuation and thickness of the patient and the presence of table, pad, and head support. The larger relative difference observed in data from protocol 1 is due to its absence of copper filtration and reduced beam quality (half-value layer), decreasing its penetrating power and augmenting the impact of patient attenuation.

Effective dose as a function of projection angle is shown in Fig 3. All individual rotations are shown and follow a similar distribution as in Fig 2. The variation observed within each curve is indicative of deviation caused by the Monte Carlo process. The relative variability in protocol 1 was due to differences in patient head size, age, attenuation, and beam quality described earlier.

Summing across individual curves shown in Fig 3 determines the effective dose for each 3D rotation. These data are shown in Fig 4, aggregated across protocol and summed for subtracted 
3DRA, alongside the effective dose for 2D-biplane DSA. The mean effective dose for biplane $2 \mathrm{D}$ imaging was $0.9 \mathrm{mSv}$, with a range of $0.2-1.7 \mathrm{mSv}$. This was significantly greater than the dose for subtracted 3DRA (factory: mean, $0.5 \mathrm{mSv}$; range, $0.3-0.7$ $\mathrm{mSv}$ ), customized subtracted (mean, $0.12 \mathrm{mSv}$; range, $0.10-0.13$ $\mathrm{mSv}$ ), and unsubtracted (mean, $0.06 \mathrm{mSv}$; range, $0.04-0.08 \mathrm{mSv}$ ) acquisitions. Data are summarized in Table 3.

Regarding organ dose estimates, the mean absorbed dose to the brain for biplane 2D imaging was $15 \mathrm{mGy}$, with range of 4.2-31 mGy, greater than the dose for subtracted 3DRA (factory: mean, $9.4 \mathrm{mGy}$; range, 4.1-14 mGy), customized subtracted (mean, $2.4 \mathrm{mGy}$; range, 2.1-2.7 mGy), and unsubtracted (mean, $1.2 \mathrm{mGy}$; range, $0.8-1.6 \mathrm{mGy}$ ) protocols. A similar relationship was calculated for active bone marrow, with mean absorbed dose estimates for $2 \mathrm{D}$ imaging $=2.1 \mathrm{mGy}$, subtracted $3 \mathrm{DRA}=1.5$ $\mathrm{mGy}$, customized subtracted 3DRA $=0.3 \mathrm{mGy}$, and unsubtracted 3DRA $=0.2 \mathrm{mGy}$. Because the thyroid was outside the primary FOV in all acquisitions, mean doses were low, with 2D imaging

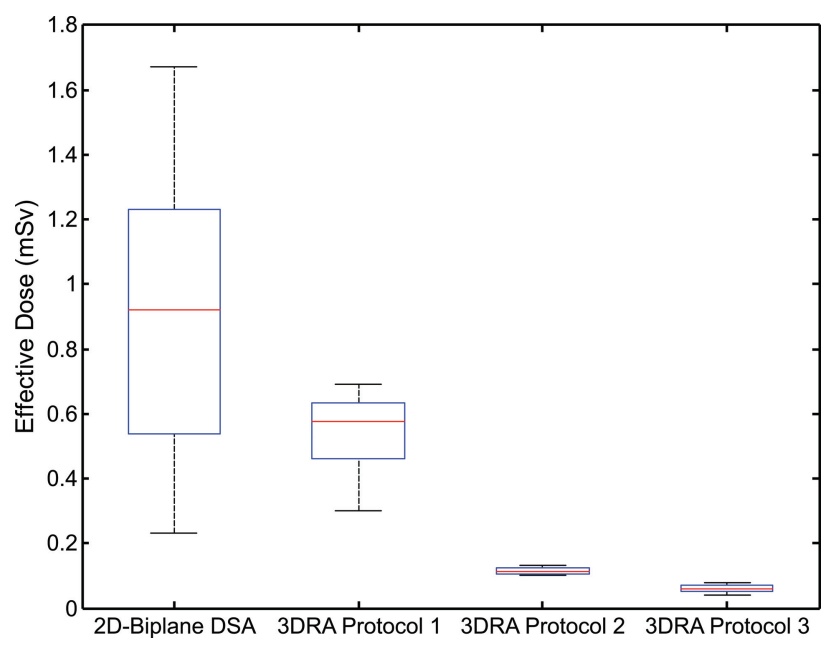

FIG 4. Box-and-whisker plot depicting the effective dose of biplane DSA and 3DRA protocols across all patients. Boxes denote median, lower, and upper quartiles, with whiskers capturing values outside the interquartile range.

Table 3: Mean, median, and range of effective dose as a function of acquisition type and protocol

\begin{tabular}{lllc}
\hline & \multicolumn{3}{c}{ Effective Dose (mSv) } \\
\cline { 2 - 4 } & Mean & Median & Range \\
\hline 2D & 0.89 & 0.92 & $0.23-1.7$ \\
Biplane DSA & & & \\
3D & 0.54 & 0.57 & $0.3-0.69$ \\
Protocol 1 & 0.12 & 0.11 & $0.1-0.13$ \\
Protocol 2 & 0.06 & 0.06 & $0.04-0.08$ \\
Protocol 3 & & & \\
\hline
\end{tabular}

Table 4: Relative effective dose comparison indicating mean, median, and range for direct and normalized comparisons

\begin{tabular}{|c|c|c|c|c|c|c|}
\hline & \multicolumn{6}{|c|}{ Relative Effective Dose } \\
\hline & \multicolumn{3}{|c|}{$E_{3 D R A} / E_{2 D-D S A}(\%)$} & \multicolumn{3}{|c|}{$\left(E_{3 D R A} / E_{2 D-D S A}\right)_{\text {Normalized }}(\%)$} \\
\hline & Mean & Median & Range & Mean & Median & Range \\
\hline Protocol 1 & 68 & 65 & $27-127$ & 61 & 67 & 25-103 \\
\hline Protocol 2 & 15 & 13 & $10-24$ & 14 & 13 & $7.4-23$ \\
\hline Protocol 3 & 8.9 & 6.1 & $3.3-29$ & 7.5 & 6.8 & $3.4-12$ \\
\hline
\end{tabular}

delivering $1.2 \mathrm{mGy}$ and $3 \mathrm{D}$ acquisitions delivering $0.3,0.1$, and $0.06 \mathrm{mGy}$ across protocols 1,2 , and 3 , respectively.

One source of variability in the $2 \mathrm{D}$-biplane data was the duration of each acquisition (total number of DSA frames), because it was free to vary depending on clinical needs. To account for this, the $2 \mathrm{D}$ data were also normalized to a run length of 60 frames (10 seconds), the median number of frames considering all DSA acquisitions (mean, $58.4 \pm 17$ frames).

A limitation of evaluating aggregate effective doses across all subjects is that the Monte Carlo model accounted for differences in reference person (patient) age. Thus, the same 2D/3D protocol applied to a 1-year-old patient would deliver a different effective dose than that delivered to a 15 -year-old patient, including adjustments for body habitus. This source of variability was removed by assessing the relative effective dose between 2D-biplane and $3 \mathrm{D}$ acquisitions in the same patient, in acquisitions evaluating the same vessel. This comparison delivers the formative conclusion of this work, illustrated in Table 4 and Fig 5: Customized 3DRA protocols delivered a mean of 9\% (range, 3\%-29\%, unsubtracted) and $15 \%$ (range, $10 \%-24 \%$, subtracted), the effective dose of a single 2D-biplane acquisition in the same vessel. The original subtracted protocol delivered a mean of $68 \%$, ranging between $27 \%$ and $127 \%$ compared with $2 \mathrm{D}$-biplane DSA. Figure 5 also compares the 3DRA imaging mode with normalized 2D-DSA, generalizing the results and reducing ranges to $3 \%-12 \%, 7 \%-22 \%$, and $25 \%-103 \%$ for the customized unsubtracted, customized subtracted, and original subtracted protocols, respectively.

\section{DISCUSSION}

Patient-centric radiation dose assessments in interventional radiology are primarily focused on the calculation of peak skin dose. ${ }^{20-22}$ Although a valuable indicator of the potential for a deterministic effect (tissue reaction), skin dose is rarely the dosimetric focus in a pediatric environment due to the greater likelihood of small patient habitus and considerably lower $\mathrm{K}_{\mathrm{a}, \mathrm{r}}$ across all procedure categories. The relevant radiation concern in pediatric interventions is largely the potential stochastic effect and the current understanding that the risk in younger patients is greater than in adults. ${ }^{19,23}$ Unlike in CT, quantifying the radiation burden in angiographic procedures is a difficult and protracted process. Many variables complicate dosimetry because the irradiated anatomy, geometry (positions of tubes, patient, table), acquisition type (fluoroscopy, fluorography, conebeam $\mathrm{CT}$ ), and technical factors (choice of imaging protocol, automatic dose rate control) can vary throughout a procedure and can differ among cases.

Comparing measured and reported radiation dose surrogates is a common practice in fluoroscopic and interventional applications. ${ }^{24-26}$ There is considerable utility in capturing and analyzing these metrics for use in quality programs and to establish notification and substantial radiation dose levels. ${ }^{27}$ Assessing the impact of $\mathrm{K}_{\mathrm{a}, \mathrm{r}}$ and air kerma area product between interventions can be complicated as it does not account for geometric factors, colli- 


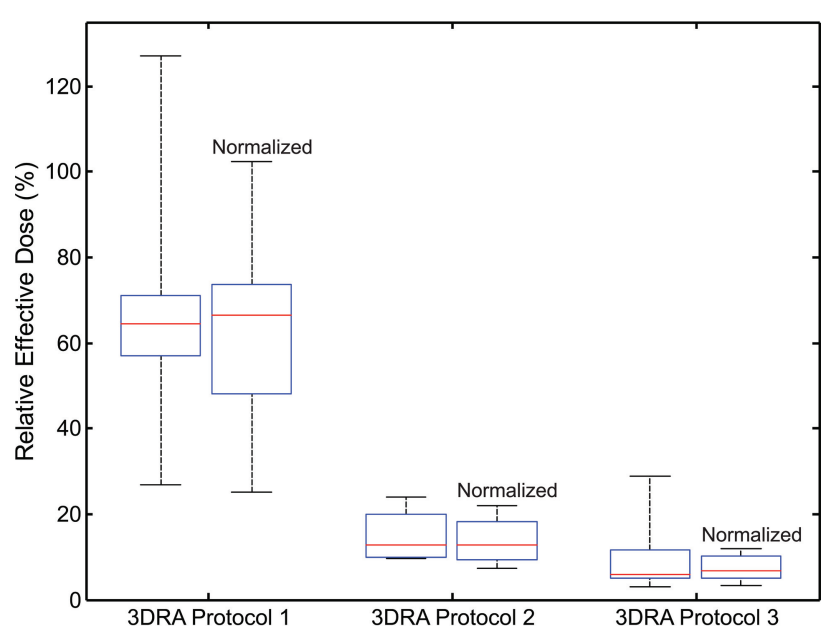

FIG 5. Box-and-whisker plot depicting the relative effective dose comparing 3DRA and biplane DSA acquisitions in the same patient. Relative comparison with the original 2D-DSA effective dose (left) and normalized DSA effective dose (right) for each 3DRA protocol. Boxes denote median, lower, and upper quartiles, with whiskers capturing values outside the interquartile range. mation, obstacles in the path of radiation (table, pad, head support, etc) and differences in beam quality. Although these are important dosimetric metrics, they cannot provide a comprehensive assessment of radiation burden in pediatric imaging.

Effective dose is a mathematic, calculated quantity intended to act as a surrogate of risk, reflecting the detriment of a nonuniform exposure in terms of an equivalent whole-body exposure. ${ }^{19} \mathrm{Be}-$ cause there are numerous techniques, computational steps, and variables in calculating the effective dose, values can vary by up to $\geq 100 \%$, depending on the applied Monte Carlo model, input data, assumptions, and approximations. ${ }^{28}$ Also, because estimates of biologic risk have evolved since the inception of effective dose, the concept of a "true" value is not accurate. Comparing effective doses using the same methods and calculation steps, however, allows a relative assessment of imaging events similar to those we investigated in this work. Although effective dose does not estimate the risk for individual patients, this work has value as part of an optimization process in pediatric neuroangiography. The number of significant figures described in Table 3 does not indicate precision and was not proposed to illustrate a difference in radiation risk but was intended to emphasize the dosimetric
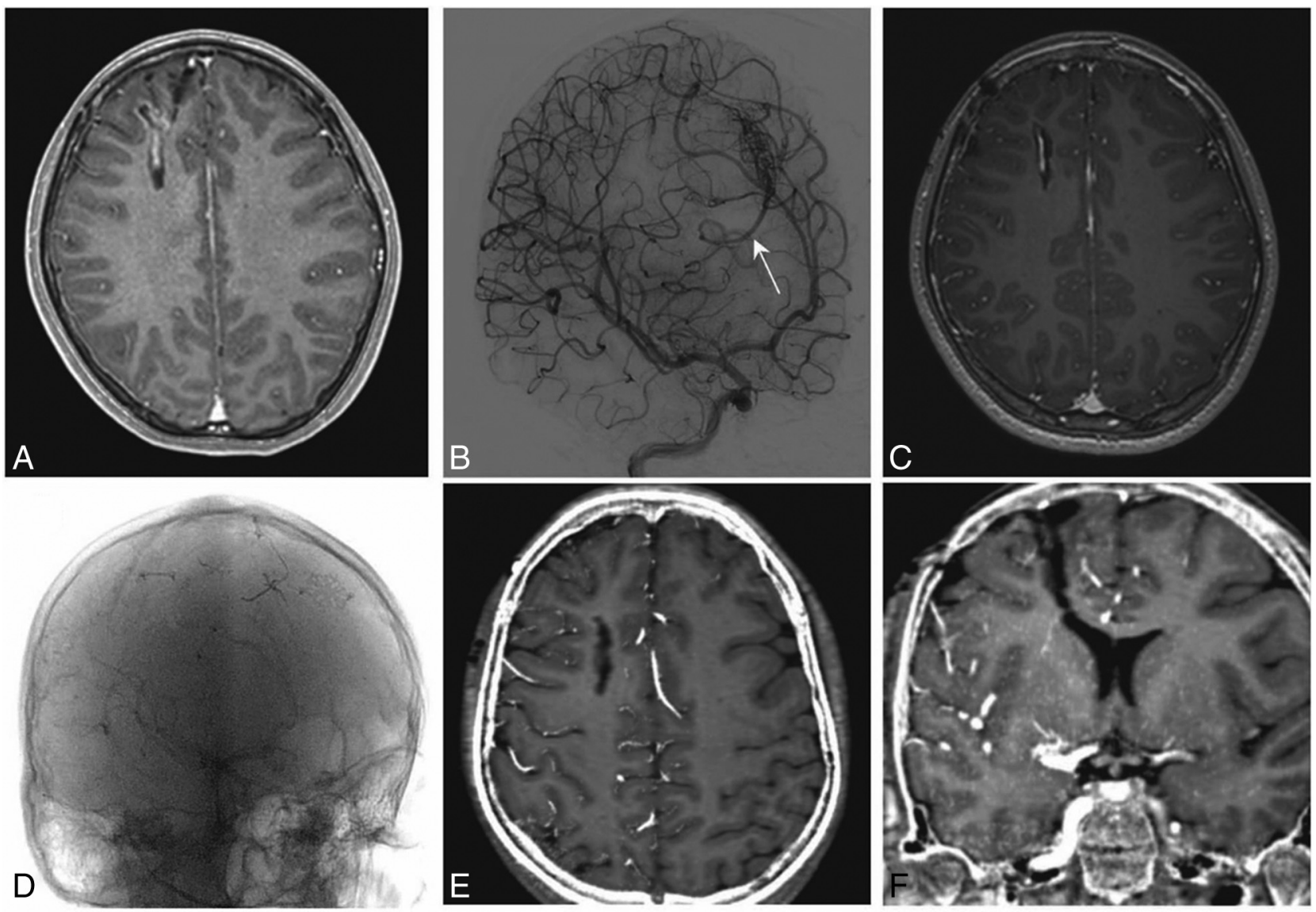

FIG 6. An example of the use of a 3D rotational angiographic sequence (protocol 3). A 12-year-old girl was referred from an outside institution 4 months after hemorrhage from a right frontal arteriovenous malformation. A, Postgadolinium axial 3D T7-weighted MR image shows the AVM nidus within a collapsed hemorrhagic cavity in the right superior frontal gyrus. $B$, Diagnostic angiography in the right internal cerebral artery in an oblique frontal projection shows the nidus fed from posterior internal frontal branches of the anterior cerebral artery, with early filling of an inferiorly draining cortical vein (arrow). The AVM was surgically resected, following which DSA (not shown) confirmed no residual nidus. C, Postgadolinium axial 3D T1-weighted MR image obtained at 6-month follow-up shows linear enhancement within the surgical cavity. Diagnostic angiography subsequently performed showed no arteriovenous shunting. $D, 3 D$ rotational angiography performed in the right internal cerebral artery using protocol 3 with $30 \%$ contrast concentration, subsequently reconstructed into CTA. $E$ and $F, 3 D$ rotational angiography fused with the pregadolinium 3D T1-weighted MR imaging sequence, which confirmed no vascular structures within the surgical cavity in multiple planes. 
differences in each acquisition protocol and the relative difference within specific procedures and imaging events. A similar limitation is appropriate for interpretation of the organ doses presented.

There has been a tendency to avoid performing 3DRA in children because the magnitude of its radiation use is not often fully understood. This has encouraged neuroradiologists to continue with their established standard of care (ie, multiple 2D-biplane DSA projections) in situations in which 3DRA may have been a viable and effective alternative. This work was intended to quantify and compare the dose differences between 2D and 3D acquisitions and to illustrate that the effective dose for a single 3DRA acquisition could be considerably lower than that for a typical biplane fluorographic acquisition and can be further reduced without loss of clinical utility though protocol customization. This work clearly illustrated that customized unsubtracted and subtracted $3 \mathrm{D}$ protocols deliver a fraction of the radiation dose delivered in a biplane $2 \mathrm{D}$ acquisition.

This work was one of many endeavors to better understand the application of radiation in 3D acquisitions in pediatric neuroangiographic practice. All 3DRA acquisitions reported in this article were of diagnostic quality and appropriate for clinical management of each patient, as illustrated in Fig 6. Qualitative assessments were made throughout the tuning process, and future work will measure the impact of varied 3D acquisition parameters on quantitative image quality in patients and phantoms. Although image quality is intricately linked to the conclusions of this article, the intention of this work was to determine the effective dose of customized and factory protocols acquired on patients at our institution. Additional research will evaluate the procedural impact of 3DRA in reducing the amount of fluorography in individual cases, assess the effects of varied proprietary postprocessing capabilities on quality and image acquisition, and further investigate the impact of 3DRA on patient management and outcomes.

\section{CONCLUSIONS}

An essential responsibility of the pediatric neuroangiographer is the knowledge of radiation dose for individual actions within the interventional suite. This includes the relative magnitude of conventional DSA and 3DRA acquisition techniques. With customization of 3D acquisition protocols, the effective dose for 3DRA can be significantly lower than for 2D-biplane imaging, solidifying its place as an essential adjunct in the evaluation of neurovascular lesions.

\section{ACKNOWLEDGMENTS}

We acknowledge Dr Timo Krings, Dr Vitor Pereira, Dr Peter Dirks, Dr James Drake, Vanessa Rea, and Evan Kitamura for their contributions to this work.

\section{REFERENCES}

1. Honarmand A, Gemmete J, Hurley M, et al. Adjunctive value of intra-arterial cone beam CT angiography relative to DSA in the evaluation of cranial and spinal arteriovenous fistulas. J Neurointerv Surg 2014;7:517-23 CrossRef Medline

2. Safain M, Rahal J, Patel S, et al. Superior performance of conebeam CT angiography in characterization of intracranial atherosclerosis. J Neurosurg 2014;121:441-49 CrossRef Medline

3. Schulz C, Schmitt M, Böckler D, et al. Intraoperative contrast-enhanced cone beam computed tomography to assess technical suc- cess during endovascular aneurysm repair. J Vasc Surg 2016;64: 577-84 CrossRef Medline

4. Patel N, Gounis M, Wakhloo A, et al. Contrast-enhanced angiographic cone-beam CT of cerebrovascular stents: experimental optimization and clinical application. AJNR Am J Neuroradiol 2011;32: 137-44 CrossRef Medline

5. Namba K, Niimi Y, Song J, et al. Use of Dyna-CT angiography in neuroendovascular decision-making: a case report. Interv Neuroradiol 2009;15:67-72 CrossRef Medline

6. Orth R, Wallace M, Kuo M; Technology Assessment Committee of the Society of Interventional Radiology. C-arm cone-beam CT: general principles and technical considerations for use in interventional radiology. J Vasc Interv Radiol 2009;20:S538-44 CrossRef Medline

7. Muthusami P, Shkumat N, Rea V, et al. CT reconstruction and MRI fusion of $3 \mathrm{D}$ rotational angiography in the evaluation of pediatric cerebrovascular lesions. Neuroradiology 2017;59:625-33 CrossRef Medline

8. Wallace M, Kuo M, Glaiberman C, et al; Technology Assessment Committee of the Society of Interventional Radiology. Three-dimensional C-arm cone-beam CT: applications in the interventional suite. J Vasc Interv Radiol 2008;19:799-813 CrossRef Medline

9. Thierry-Chef I, Simon S, Miller D. Radiation dose and cancer risk among pediatric patients undergoing interventional neuroradiology procedures. Pediatr Radiol 2006;36:159-62 CrossRef Medline

10. Orbach D, Stamoulis C, Strauss K, et al. Neurointerventions in children: radiation exposure and its import. AJNR Am J Neuroradiol 2014;35:650-56 CrossRef Medline

11. Wielandts J, De Buck S, Ector J, et al. Three-dimensional cardiac rotational angiography: effective radiation dose and image quality implications. Europace 2010;12:194-201 CrossRef Medline

12. Peters M, Krings G, Koster M, et al. Effective radiation dosage of three-dimensional rotational angiography in children. Europace 2015;17:611-16 CrossRef Medline

13. Kim S, Yoshizumi T, Frush D, et al. Radiation dose from cone beam $\mathrm{CT}$ in a pediatric phantom: risk estimation of cancer incidence. $A J R$ Am J Roentgenol 2010;194:186-90 CrossRef Medline

14. Wang C, Nguyen G, Toncheva G, et al. Evaluation of patient effective dose of neurovascular imaging protocols for C-arm cone-beam CT. AJR Am J Roentgenol 2014;202:1072-77 CrossRef Medline

15. Wielandts J, Smans K, Ector J, et al. Effective dose analysis of threedimensional rotational angiography during catheter ablation procedures. Phys Med Biol 2010;55:563-79 CrossRef Medline

16. Bai M, Liu X, Liu B. Effective patient dose during neuroradiological C-arm CT procedures. Diagn Interv Radiol 2013;19:29-32 CrossRef Medline

17. Lin P, Schueler B, Balter S, et al. Accuracy and calibration of integrated radiation output indicators in diagnostic radiology: a report of the AAPM Imaging Physics Committee Task Group 190. Med Phys 2015;42:6815-29 CrossRef Medline

18. Tapiovaara M, Siiskonen T. PCXMC: a Monte Carlo program for calculating patient doses in medical $\mathrm{x}$-ray examinations. Helsinki: STUK; 2008

19. The 2007 Recommendations of the International Commission on Radiological Protection. Ann ICRP 2007;37:1-332 CrossRef Medline

20. Khodadadegan $Y$, Zhang M, Pavlicek W, et al. Validation and initial clinical use of automatic peak skin dose localization with fluoroscopic and interventional procedures. Radiology 2013;266:246-55 CrossRef Medline

21. Kwon D, Little M, Miller D. Reference air kerma and kerma-area product as estimators of peak skin dose for fluoroscopically guided interventions. Med Phys 2011;38:4196-204 CrossRef Medline

22. Vano E, Fernandez J, Sanchez R, et al. Patient radiation dose management in the follow-up of potential skin injuries in neuroradiology. AJNR Am J Neuroradiol 2013;34:277-82 CrossRef Medline

23. Committee to Assess Health Risks from Exposure to Low Levels of Ionizing Radiation. Health Risks from Exposure to Low Levels of Ioniz- 
ing Radiation: BEIR VII Phase 2. Washington, DC: National Academies Press; 2006

24. Chida K, Saito H, Otani H, et al. Relationship between fluoroscopic time, dose-area product, body weight, and maximum radiation skin dose in cardiac interventional procedures. AJR Am J Roentgenol 2006;186:774-78 CrossRef Medline

25. Chida K, Ohno T, Kakizaki S, et al. Radiation dose to the pediatric cardiac catheterization and intervention patient. AJR Am J Roentgenol 2010;195:1175-79 CrossRef Medline
26. Manica J, Borges M, Medeiros R, et al. A comparison of radiation dose between standard and 3D angiography in congenital heart disease [in English, Portuguese]. Arg Bras Cardiol 2014;103:131-37 Medline

27. Radiation Dose Management for Fluoroscopically Guided Interventional Medical Procedures. Bethesda: National Council on Radiation Protection and Measurements. Report No. 1682010

28. McCollough C, Christner J, Kofler J. How effective is effective dose as a predictor of radiation risk? AJR Am J Roentgenol 2010;194:890-96 CrossRef Medline 